\title{
Trigeminocardiac reflex during surgery in the cerebellopontine angle
}

\section{Bernhard Schall, M.D., Rudolf Probst, M.D., Stephan Strebel, M.D., Peter Fuhr, M.D. and Otmar Gratzl, M.D.}

Departments of Neurological Surgery, Otorhinolaryngology, Anaesthesia, and Neurology, University Hospitals Basel, Basel, Switzerland

In different experimental studies authors have analyzed the autonomic responses elicited by the electrical, mechanical, or chemical stimulation of the trigeminal nerve system. The trigeminocardiac reflex (TCR) is a well-recognized phenomenon that consists of bradycardia, arterial hypotension, apnea, and gastric hypermotility. It occurs during ocular surgery and during other manipulations in and around the orbit. Thus far, it has not been shown that central stimulation of the trigeminal nerve can also cause this reflex.

The TCR was defined as clinical hypotension with a drop in mean arterial blood pressure (MABP) of more than $20 \%$ and bradycardia lower than 60 beats/minute. Pre-, intra-, and postoperative heart rate (HR) and blood pressure were reviewed retrospectively in 125 patients who underwent surgery for tumors of the cerebellopontine angle (CPA), and they were divided into a TCR group and a non-TCR group. Of the 125 patients, $14(11 \%)$ showed evidence of TCR during dissection of the tumor near the trigeminal nerve at the brainstem. Their HR fell $38 \%$ and their MABP fell $48 \%$ during operative procedures as compared with preoperative levels. After cessation of manipulation, the HR and the MABP returned to preoperative levels. Risk factors for the occurrence of TCR were compared with results from the literature.

The authors' results show the possibility of occurrence of a TCR during manipulation of the central part of the trigeminal nerve when performing surgery in the CPA.

Key Words * cerebellopontine angle * trigeminocardiac reflex * trigeminal nerve * bradycardia * hypotension

The trigeminal nerve is the largest of the cranial nerves, and it provides primarily sensory supply to the face, scalp, and mucosa of the nose and mouth. Stimulation of the trigeminal receptors that innervate the nose and nasal passages is thought to provide the most important afferent input for initiation of the oculocardiac reflex (OCR) and trigeminorespiratory reflex, which were first described in the cat and the rabbit.[20] These reflexes are well-recognized phenomena consisting of bradycardia, arterial hypotension, apnea, and/or gastric hypermotility. In 1969 and 1975, Angell-James and Daly[4,5] 
confirmed that in dogs, after induction of pentobarbital or chloralose-urethane anesthesia, stimulation of the nasal mucosal membrane (with cold water, tabacco smoke, or an electrical square-wave stimulus of 2-5 V, 1-msec duration, and 10-20 Hz) caused bradyapnea and/or expiratory apnea with bradycardia and variable changes in blood pressure. All these responses were abolished by induction of local anaesthesia to the nose, which forms the afferent trigeminal pathway for such reflexes. Different surgical procedures requiring manipulation of the trigeminal ganglion and roots are known to lead to autonomic changes such as bradycardia or asystolia. The OCR, a variant of the trigeminocardiac reflex (TCR), is a well-recognized phenomenon occurring during ocular surgery,[10,12] but also during other manipulations in and around the orbit.[2,15,18,30,35,43] In 1987, it was suggested that the TCR could be elicited by stimulating the afferent pathway not only from the ciliary nerves but also from the sensory branches of the maxillary and mandibular divisions of the trigeminal nerve.[7,25] Eighteen cases of induced TCR during corrective surgery for craniofacial and maxillofacial deformities have been described.[7,8,17,23,25,33,35,39,40,43] During the last few years, different authors who performed percutaneous compression of the trigeminal ganglion have reported the occurrence of significant autonomic changes coinciding with foramen ovale puncture and ganglion compression, $[9,14,16,22,29,41]$ but no clear connection to TCR has been made.

To the best of our knowledge, we present the first systematic study that provides evidence of the TCR during manipulation of the central part of the trigeminal nerve during surgery in the cerebellopontine angle (CPA).

\section{CLINICAL MATERIAL AND METHODS}

We defined the occurrence of TCR as clinical hypotension with a drop in mean arterial blood pressure (MABP) of $20 \%$ and bradycardia lower than 60 beats/minute: cessation of traction must have resulted in a spontaneous increase of the heart rate (HR) and the MABP to normal levels. The phenomenon had to recur when traction was repeated.

\section{Patient Population}

We retrospectively reviewed the case histories of 125 patients who underwent surgery for tumors of the CPA performed by an otoneurosurgical team from January 1988 to February 1998 at the University Hospital, Basel, Switzerland. Of these 125 patients, 14 (11\%) showed evidence of TCR during tumor dissection near the trigeminal nerve at the brainstem. The TCR group included nine women and five men, and their average age was 44 years (standard deviation [SD] 11 years, range 14-72 years). The non-TCR group included 58 women and 53 men, and their average age was 51 years (SD 14 years, range 34-75 years). Preoperatively, all 125 patients underwent vestibular testing (eye-tracking test, optokinetic reflex, vestibuloocular reflex), audiological testing (pure tone audiogram, speech discrimination testing, and brainstem auditory evoked potentials), and neuroradiological examinations (cerebral computerized tomography scanning, cerebral magnetic resonance imaging or digital subtraction arteriography of cerebral blood vessels).

In the 14 patients showing evidence of TCR, tumor size was measured as the largest tumor diameter and categorized as medium (20-30 mm; six patients [43\%]) or large (> $30 \mathrm{~mm}$; eight patients [57\%]). The average tumor size was $35 \mathrm{~mm}$ (SD $10 \mathrm{~mm}$, range 22-50 mm). Tumors were classified according to the World Health Organization histological typing of central nervous system tumors.[27] There were 10 vestibular schwannomas (six Antoni Type A, four Antoni Type B) and four meningiomas (two psammomatous, one meningothelial, one fibroplastic). 


\section{Surgical Technique, Intra-, and Perioperative Management}

A retrosigmoid approach with the patient in the supine position was used in all surgical procedures. The surgical technique has been described previously.[31] Intraoperative nerve-integrity monitoring of the cochlear nerve (Nicolet Compact Four; Nicolet Instruments, Madison, WI) and the facial nerve (Neurosign 100; Maxtim, Germany) was conducted routinely. Intracranial dissection was performed using a surgical microscope (Zeiss/Contraves; Carl Zeiss, Inc, Wetzikon, Switzerland). After surgery, patients were monitored in the intensive care unit for the first 24 hours and in the neurosurgical recovery room for the next 24 to 72 hours.

\section{Anesthetic Technique}

Patients fasted for at least 6 hours and were premedicated orally with midazolam prior to surgery. Routine monitoring during surgery included electrocardiography, HR (72 beats/minute, SD 10, range 58-89); end-tidal concentration of carbon dioxide $\left(\mathrm{P}_{\mathrm{ET}} \mathrm{CO}_{2}\right.$; range 3.8-4.1 kPA) and isoflurane ( $\left.\mathrm{P}_{\mathrm{ET}} \mathrm{ISO}\right)$; pulse oximetry (oxygen saturations $>96 \%$ ); and esophageal temperature (Spacelabs, Redmond, WA). An indwelling radial artery catheter was inserted to allow continuous invasive MABP ( $83 \mathrm{~mm} \mathrm{Hg}, \mathrm{SD} 13$, range 72-142) measurements and intermittent blood gas samples. All hemodynamic parameters were monitored continuously and recorded throughout the surgical procedure.

Anesthesia was induced with thiopental 3 to $4 \mathrm{mg} / \mathrm{kg}^{-1}$, followed by fentanyl $3 \mathrm{mg} / \mathrm{kg}^{-1}$, and atracurium $0.5 \mathrm{mg} / \mathrm{kg}^{-1}$. After the trachea was intubated, the lungs were mechanically ventilated (Sulla $808 \mathrm{~V}$; Lübeck, Germany) with a mixture of air and oxygen $\left(\mathrm{F}_{\mathrm{I}} \mathrm{O}_{2}=0.5\right)$. Anesthesia was maintained with isoflurane 1.2 ( $\mathrm{P}_{\mathrm{ET}} \mathrm{ISO} 74 \%$, SD 19, range 55-110), and when it seemed clinically necessary, an additional bolus of fentanyl and atracurium was administered.

\section{Statistical Analysis}

All statistical analyses were performed using statistical software (STATView II 1.01; Abacus Concepts, Inc, Berkeley, CA) on a commercially available computer. Data are presented as the mean \pm SD unless otherwise indicated. To compare two independent proportions, Fisher's exact test was used. The level of significance was set at $\mathrm{p}$ less than 0.05 .

\section{RESULTS}

Fourteen patients met the inclusion criteria. There were no significant differences in the age, sex, histological composition of the tumor, or duration and distribution of symptoms between the TCR and the non-TCR groups. Possible risk factors for the 14 patients compared to non-TCR patients are summarized in Table 1. 


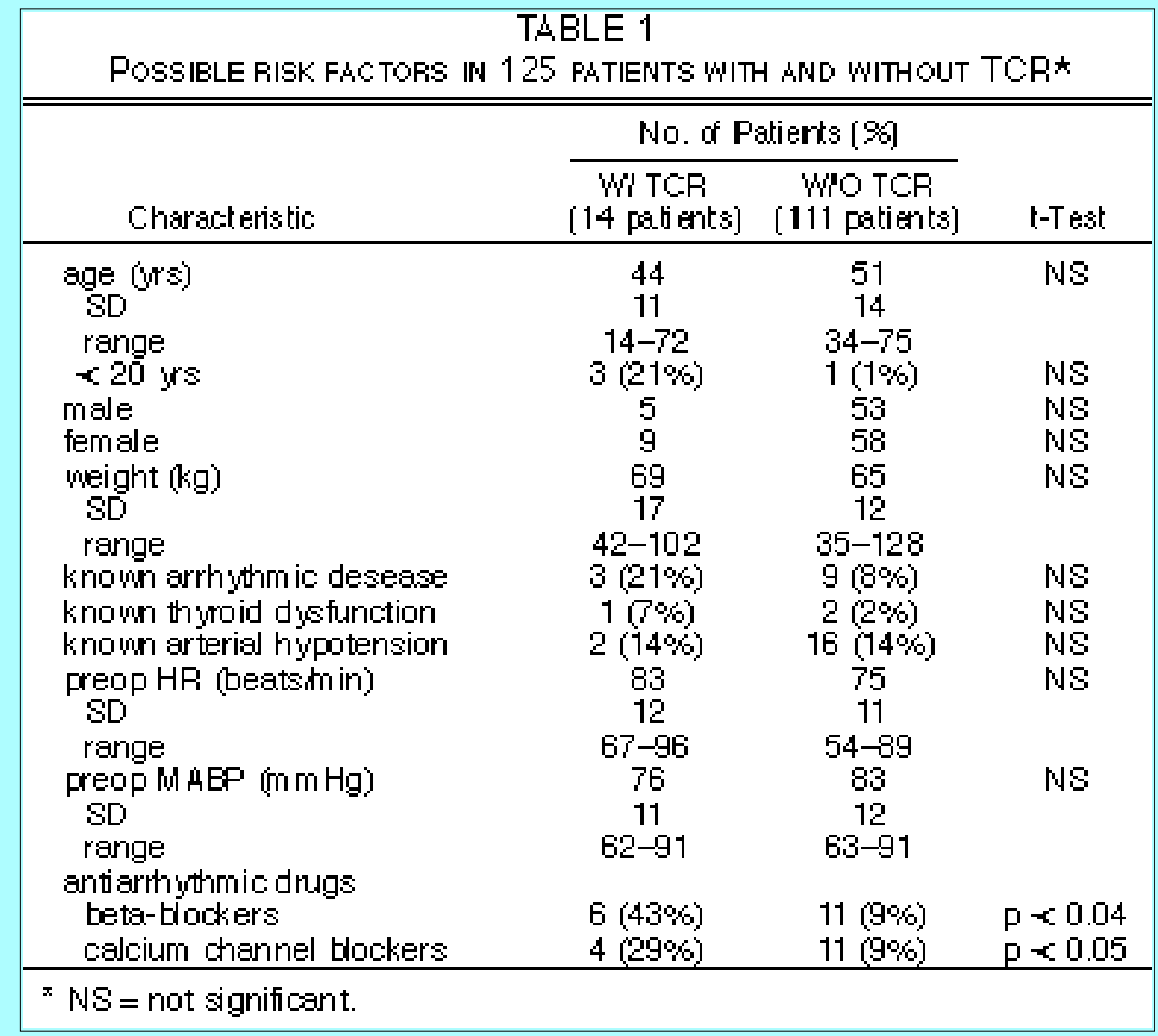

During dissection of the tumor near the trigeminal nerve at the brainstem, the patient's HR and MABP decreased significantly, as defined in the inclusion criteria. The mean HR fell $38 \%$ from a mean of 76 beats/minutes (SD 11 beats/minute, range 62-91) before manipulation to a mean of 47 beats/minute (SD 12 beats/minute, range 20-59) during the procedure and returned to a mean of 77 beats/minute (SD 9 beats/minute, range 65-93) after manipulation (Fig. 1).

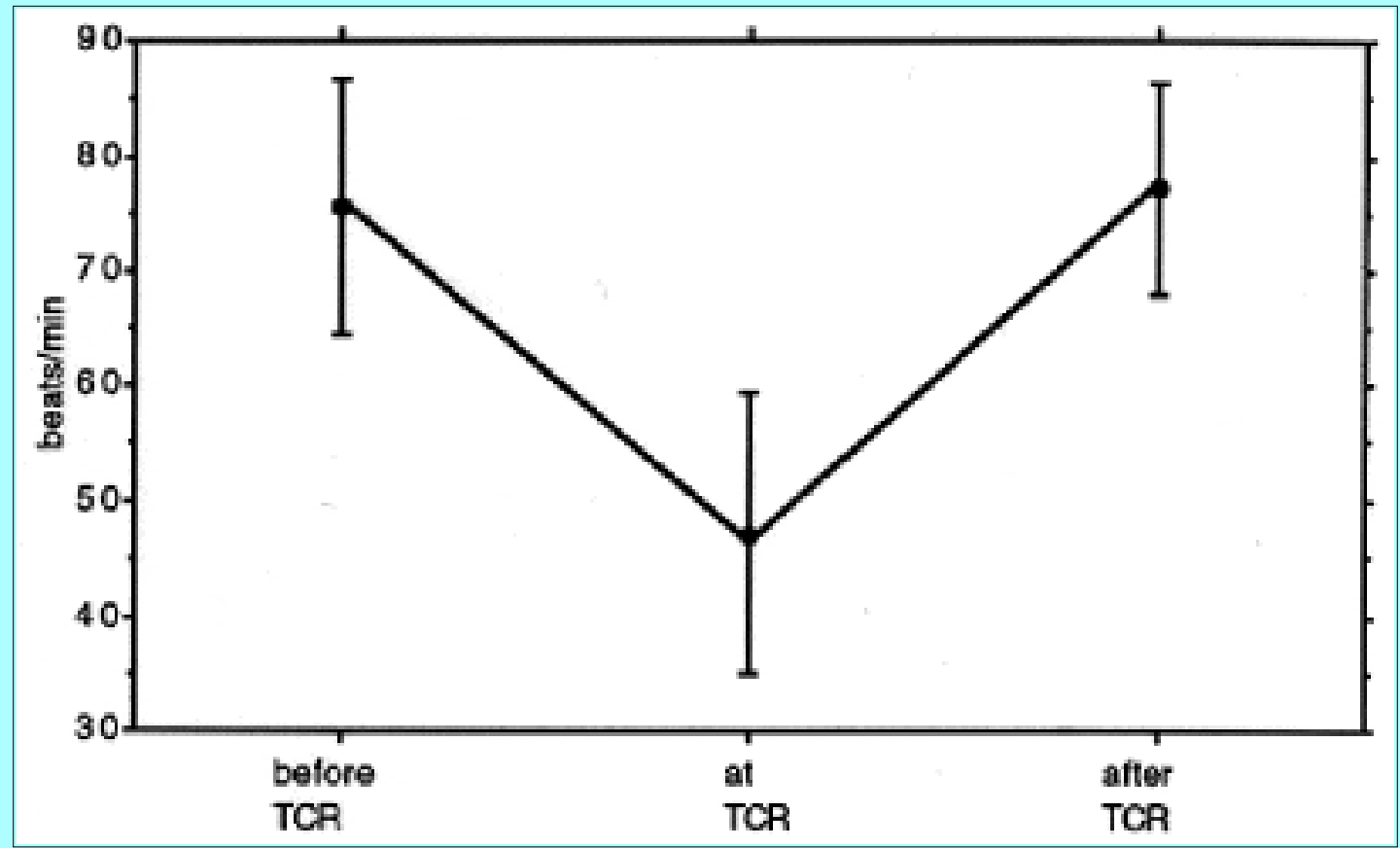

Fig. 1. Graph depicting the HR before and during the occurrence of TCR in 14 patients. 
Values expressed as the mean \pm SD.

The MABP fell $48 \%$ from a mean of $84 \mathrm{~mm} \mathrm{Hg}$ (SD $7 \mathrm{~mm} \mathrm{Hg}$, range 71-93) before manipulation to 44 $\mathrm{mm} \mathrm{Hg}$ (SD $12 \mathrm{~mm} \mathrm{Hg}$, range 10-56) during the procedure, and returned to $82 \mathrm{~mm} \mathrm{Hg}$ (SD $8 \mathrm{~mm} \mathrm{Hg}$, range 69-95) after manipulation (Fig. 2). The HR and MABP after manipulation were not significantly different from before. In three cases, there was an asystolia, with a duration between 30 to 70 seconds.

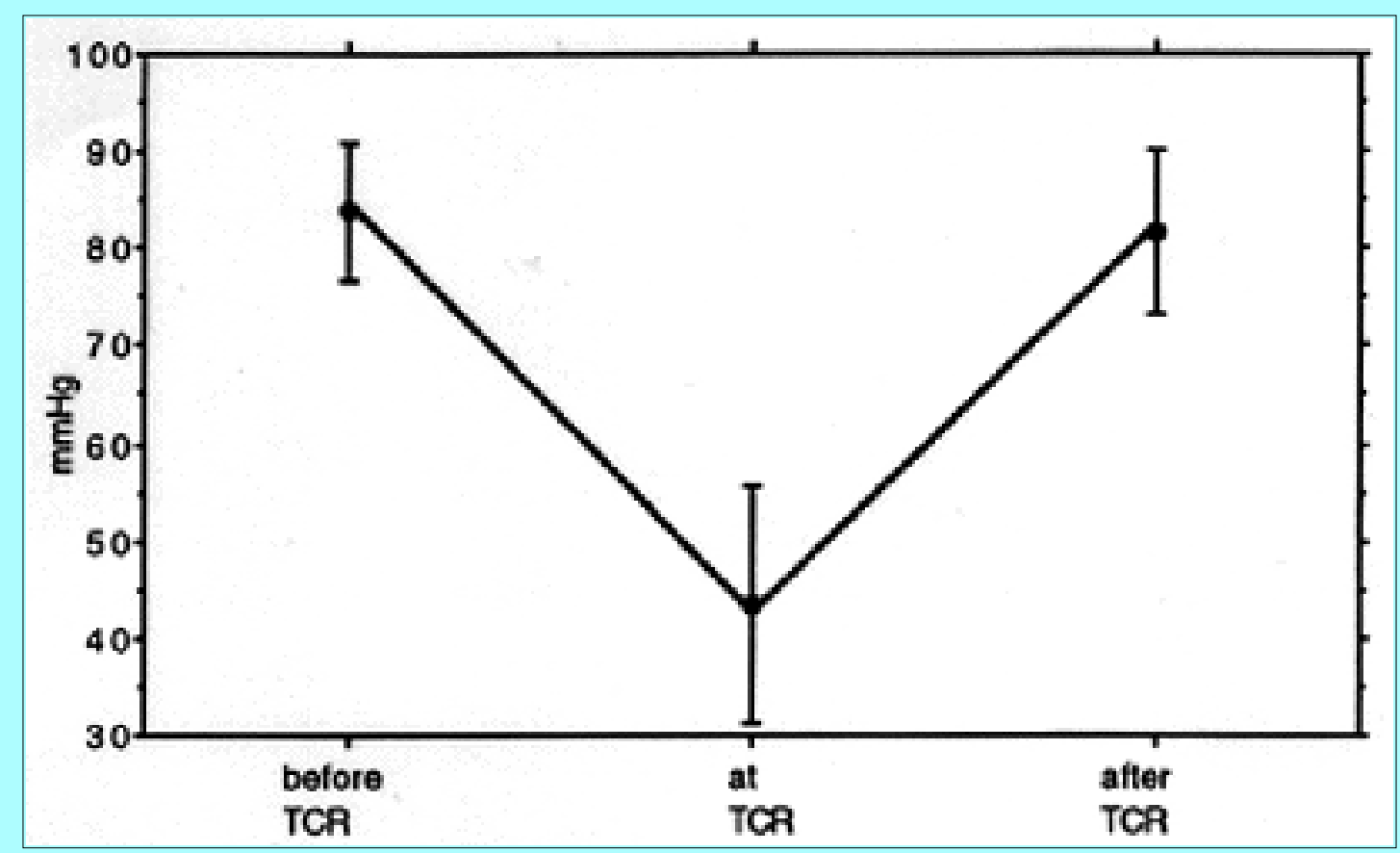

Fig. 2. Graph showing the MABP before and during the occurrence of TCR in 14 patients. Values expressed as the mean \pm SD.

Intravenous administration of atropine $(0.9 \mathrm{mg}$; SD $2 \mathrm{mg}$, range $0.6-1.2 \mathrm{mg}$ ) lead to a cessation of the TCR during the remainder of the surgical procedure in all 14 cases. Routine electrocardiographic monitoring during follow-up examination in the intensive care unit after surgery showed a new intermittent nodal rhythm (sinus arrhythmia) in one patient. Further complications, which could be attributed to the occurrence of TCR, were not detected.

\section{DISCUSSION}

In various experimental studies authors have analyzed the autonomic responses elicited by the electrical, mechanical, or chemical stimulation of the trigeminal nerve system.[4,5,20,45] One variant of the different trigeminal reflexes, TCR is a well-recognized phenomenon that consists classically of bradycardia, arterial hypotension, apnea, and gastric hypermotility.[21] Different variants of TCR exhibit their own characteristics. The OCR is associated with bradycardia but not arterial hypotension.[10,12] The diving reflex (elicited by facial immersion) and the nasopharyngeal reflex (from noxious stimulation of the nasal mucosa) are associated with an increase in peripheral vascular resistance resulting in hypertension.[21]

A uniform definition of TCR does not exists thus far; however, Bosomworth, et al.,[13] assumed a positive OCR response to be any reduction in HR of $10 \%$ or more. In the present series, we have defined TCR as the occurrence of bradycardia (HR 3/4 60 beats/minute) and a $20 \%$ or more decrease of MABP.

\section{Afferent Pathway Most Frequently Involved in TCR}


Case reports are, in general, too imprecise to allow identification of the sensory nerve involved in the afferent conduction of the TCR. Laboratory investigations, clinical experience with the TCR other than the OCR, $[7,8,17,23,25,33,35,39,40,43]$ and knowledge of the anatomical distribution of the trigeminal nerve[25,33] support the assumption that stimulation of any sensory branch of the fifth cranial nerve may lead to severe bradycardia and/or arterial hypotension[11] (Table 2). However, all previously described afferent pathways of the trigeminal nerve represent the peripheral part of the nerve. Until now, nothing has been known about the stimulation of the central part of the fifth cranial nerve.

\begin{tabular}{|c|c|c|}
\hline \multicolumn{3}{|c|}{$\begin{array}{c}\text { TABLE } 2 \\
\text { AFFERENT PATHWAYS INYOLYED IN TCRS* }\end{array}$} \\
\hline Authors \& Year & $\begin{array}{l}\text { Sensory Nerve } \\
\text { \&or Branches }\end{array}$ & $\begin{array}{l}\text { Trigeninal } \\
\text { Division } \\
\end{array}$ \\
\hline Bainton \& Liż, 1987 & zygomatiootem poral tranches & v2 \\
\hline & & \\
\hline Loewinger, et al., 1987 & zygomaticotem poral tranches & $\sqrt{2}$ \\
\hline She arer \& Wivenstone, 1987 & zygomaticotem poral tranches & $v_{2}$ \\
\hline & auricolo tem poral nerie & v3 \\
\hline Hopkins, 1988 & recurrent tentorial tranches & V1 \\
\hline $\begin{array}{l}\text { Shelly \& Church, } 1988 \\
\text { Sto tt, } 1989\end{array}$ & $\begin{array}{l}\text { maxillary ner te tranches } \\
\text { frontal supratrochlear nerves }\end{array}$ & $v_{2}$ \\
\hline & superior al veolar tranches & v2 \\
\hline $\begin{array}{l}\text { Ragno, et al., } 1989 \\
\text { Barnard \& Bainton. } 1990\end{array}$ & maxillary ner we tranches & v2 \\
\hline Barnard \& Bainton, 1990 & $\begin{array}{l}\text { maxillar y ner te tranches } \\
\text { zygomaticotem poral tranches }\end{array}$ & $v_{2}$ \\
\hline & auricolo tem poral ner'e & v3 \\
\hline Lang, et al., 1991 & $\begin{array}{l}\text { maxillary ner we tranches } \\
\text { in ferior al weolar ner'e }\end{array}$ & $v_{2}$ \\
\hline
\end{tabular}

In our cases in which TCR occurred during tumor resection in the CPA, manipulation of the trigeminal nerve at the brainstem was found to trigger the reflex. Acording to Lang, et al.,[23] the afferent pathway continues to the main sensory nucleus of the trigeminal nerve under the floor of the fourth ventricle. Short internuclear nerve fibers connect with the efferent pathway in the reticular formation, which originates in the motor nucleus of the vagus nerve.[23] Depressor fibers of the vagus nerve end in the myocardium (Fig. 3),[23] leading to autonomic changes. 


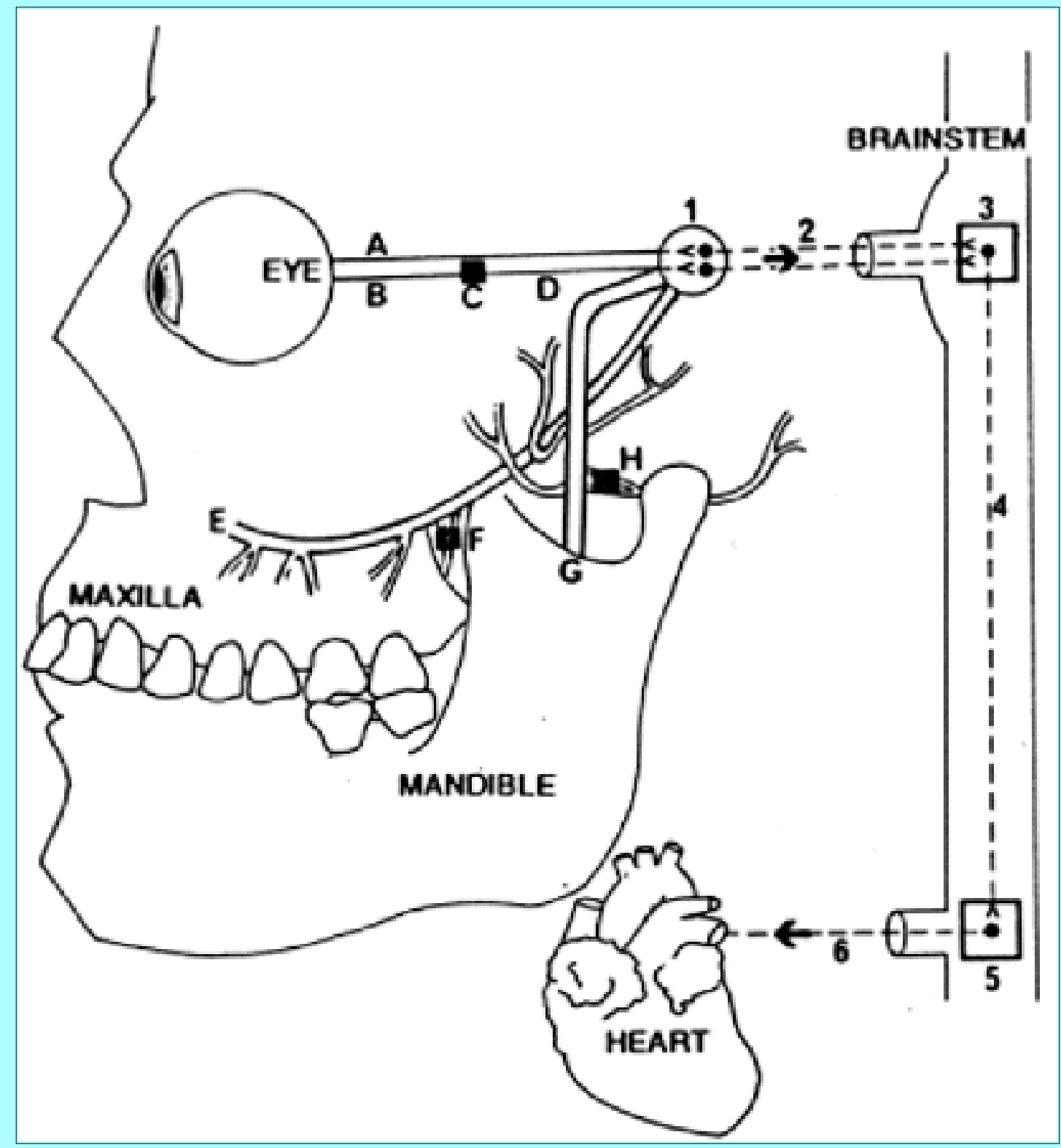

Fig. 3. Drawing illustrating the pathway of TCR. $\mathrm{A}=$ long ciliary nerve; $\mathrm{B}=$ short ciliary nerve; $\mathrm{C}=$ ciliary ganglion; $\mathrm{D}=$ ophthalmic nerve; $\mathrm{E}=$ maxillary nerve $\mathrm{F}=$ pterygopalatine ganglion; $\mathrm{G}=$ mandibular nerve; $\mathrm{H}=$ otic ganglion; 1 = gasserian ganglion; $2=$ trigeminal nerve; $3=$ sensory nucleus of trigeminal nerve; $4=$ short internucial fibers; $5=$ motor nucleus of vagus nerve; $6=$ vagus nerve. Adapted from Lang, et al. Trigemino-cardiac reflexes: maxillary and mandibular variants of the oculocardiac reflexes. Can J Anaesth 38:757-760, 1991.

A question arises in this context: is the so-called "TCR" simply a "pain response" and not a real reflex? Facial receptors may be linked to the spinal autonomic system.[14] Ruggiero, et al.,[36] have demonstrated projections from the spinal trigeminal nucleus to the entire length of the spinal cord in the rat. Menetrey and Basbaum[26] have shown in the rat extensive projection of spinal and trigeminal neurons, especially from the paratrigeminal nucleus, to the nucleus solitarii. Afferent input from the face may be transmitted to the nucleus tractus solitarii by a trigeminospinal solitary tract.[14] Part of this input may be nociceptive and generate pain-related somatovisceral and viscerovisceral reflexes.[14] To distinguish between the hypothesis of a real reflex or a pain response, we have to establish a cause-effect relationship. 


\section{Evidence of Cause-Effect Relationship}

As described by Blanc,[12] the criteria necessary to establish a cause-effect relationship include the consistency and strength of association, presence of a type of stimulus-incidence relationship, and biological plausibility. In each of the 14 cases presented in this series, the surgical manipulation of the trigeminal nerve near the brainstem elicited a specific and unequivocal effect (sudden bradycardia and arterial hypotension). Elimination of the inducing stimulus, such as manipulations near or at the trigeminal nerve, resolved the effect, and repetition of the stimulus lead each time to the same effect.

The cause-effect association can be evaluated further by examining the effectiveness of measures used to prevent the response.[11] The reflex can be prevented by avoiding stimulation of the afferent pathway, as described above, or by blocking the nerves that conduct the afferent impulses. In each of our patients, anticholinergic medication to block muscarine receptors of the heart was an effective prophylactic measure, and no further episodes of dysrhythmia were detected. The surgical procedures were completed uneventfully. In addition, administration of fentanyl to block possible pain repsonse showed no effect on further episodes of TCR.

\section{Variability of TCR}

In addition to the variety of factors that trigger TCR, its responses vary widely as well. These responses include the common sinus bradycardia with or without junctional escape, junctional rhythm, as well as other cardiac arrhythmias such as atrioventricular block, bigeminy, and nodal beats.[43] The bradycardia can progress to include sinus arrest or even asystole or ventricular fibrillation.[43] Precious, et al.,[32] have reported an incidence of $1.6 \%$ (eight of 502 cases) of either asystole or bradycardia in patients undergoing maxillofacial orthognathic or temporomandibular surgery leading back to TCR. The TCR persists for as long as the stimulus is present, but it weakens with repeated stimulation at short intervals.[15,23] The critical period occurs during the first few seconds after stimulation, when cardiac depression is maximal. Initial baseline HR has no influence on the incidence of TCR.[23] For this reason, tachycardia is not protective.[12,23]

The type of stimulus may modify the TCR response.[11] For example, as electrical stimulation of the vagus nerve is increased up to frequencies of 20 to $30 \mathrm{~Hz}$, the sinoatrial node becomes progressively more inhibited.[11] At higher frequencies, the vagal response may again decrease.[11] When the stimulation stops, the HR rapidly returns to its previous level, reflecting the rapid hydrolysis of the acetylcholine that was liberated during stimulation.[11,24] It has been shown that abrupt and sustained traction is more likely to elicit the TCR than smooth and gentle traction.[12] Terui, et al.,[45] have shown in rabbits that $\mathrm{C}$-fiber excitation in the infraorbital nerve required higher-intensity stimulation than did A-delta-fiber stimulation. Thus, stimulation sufficient to excite C-fibers leads to an overall pressor response.[45] It is expected that changing the stimulus modifies the TCR relationship although the type of stimulus is not mentioned in clinical reports dealing with TCR other than the OCR.[11]

\section{Prophylaxis and Treatment of TCR}

Because both bradycardia and hypotension may lead to hypoperfusion and cerebral or myocardial infarction, there is potential benefit from blocking the depressor response. Based on results from ocular surgery, the following factors are known to increase the risk of the TCR: hypercapnia, hypoxemia, light general anesthesia, age (more pronounced in children, perhaps due to higher resting vagal tone), the nature of the provoking stimulus (stimulus strength, duration, and waveform), and drugs.[12,23] 
Hypercapnia increases the incidence of the TCR in spontaneously breathing infants and children,[11,12] and hypoxemia must be prevented due to the possibility of cardiac arrhythmias. These ventilatory abnormalities may be due to, or aggravated by, a trigeminorespiratory reflex, which is commonly associated with TCR. They can be elicited in animals[1,4,5,20] and in humans.[12] The diving reflex in seals and other diving vertebrates results from trigeminal afferent activity.[3,44] Potent narcotic agents such as sufentanil or alfentanil, beta-blockers, and calcium channel blockers may produce predisposition to this reflex.[23,34,37,42] Narcotic agents may augment vagal tone through their inhibitory action on the sympathetic nervous system.[23]

Recognized therapeutic maneuvers include avoidance of factors known to cause predisposition for the reflex, cessation or modulation of the surgical stimulus, intravenously administered anticholinergic medication (atropine or glycopyrrolate), and local anesthetic blockade of the afferent nerves.[1,23,27,28] Once the HR has returned to normal, neural blockade and/or the administration of intravenous anticholinergics are indicated.[11] However, cholinergic blockage reduces but does not totally prevent either bradycardia or hypotension in animals, because the response includes both activation of vagal cardioinhibitory fibers and inhibition of adrenergic vasoconstriction.[21]

\section{CONCLUSIONS}

We have shown that significant decrease in HR and blood pressure occurred during CPA tumor dissection near the trigeminal nerve at the brainstem. This decrease led to TCR in up to $11 \%$ of our surgical procedures near or at the trigeminal nerve. These findings indicate, for the first time, the presence of a central induction of the TCR in humans.

We recommend that all patients undergoing tumor removal in the CPA have continuous monitoring of arterial blood pressure and HR both during surgery and for at least 24 hours after the procedure. Intravenous atropine should be available for immediate use. A noninvasive, temporary pacemaker that can respond rapidly to abrupt changes in HR should be considered for patients with known risk factors for TCR.

\section{Acknowledgment}

We thank Frances P. Harris, Ph.D., for editing the manuscript.

\section{References}

1. Alexander JP: Reflex disturbances of cardiac rhythm during ophthalmic surgery. Br J Ophthalmol 59:518-524, 1975

2. Anderson RL: The blepharocardiac reflex. Arch Ophthalmol 96:1418-1420, 1978

3. Andersen HT: Physiological adaptations in diving vertebrates. Physiol Rev 46:212-243, 1966

4. Angell-James JE, Daly M de B: Nasal reflexes. Proc R Soc Med 62:1287-1293, 1969

5. Angell-James JE, Daly MB: Some aspects of upper respiratory tract reflexes. Acta Otolaryngol 79:242-252, 1975

6. Bainton R, Lizi E: Cardiac asystole complicating zygomatic arch fracture. Oral Surg Oral Med Oral Pathol 64:24-25, 1987 
7. Bainton R, Barnard NA, Wiles JR, et al: Sinus arrest complicating a bitemporal approach to the treatment of pan-facial fractures. Br J Oral Maxillofac Surg 28:109-110, 1990

8. Barnard NA, Bainton R: Bradycardia and the trigeminal nerve. J Craniomaxillofac Surg 18:359-360, 1990

9. Belber CJ, Rak RA: Balloon compression rhizolysis in the surgical management of trigeminal neuralgia. Neurosurgery 20:908-913, 1987

10. Bietti GB: Problems of anaesthesia in strabismus surgery, in Schlomman A, Priesty BS (eds): Strabismus. Boston: Little, Brown, 1973, Vol 13, pp 727-737

11. Blanc VF: Trigeminocardiac reflexes. Can J Anaesthiol 38:696-699, 1991 (Editorial)

12. Blanc VF, Hardy JF, Milot J, et al: The oculocardiac reflex: a graphic and statistical analysis in infants and children. Can Anaesthet Soc J 30:360-369, 1983

13. Bosomworth PP, Ziegler CH, Jakoby J: The oculocardiac reflex in eye muscle surgery. Anesthesiology 19:7-10, 1958

14. Brown JA, Preul MC: Trigeminal depressor response during percutaneous microcompression of the trigeminal ganglion for trigeminal neuralgia. Neurosurgery 23:745-748, 1988

15. Chesley LD, Shapiro RD: Oculocardiac reflex during treatment of an orbital blowout fracture. J Oral Maxillofac Surg 47:522-523, 1989

16. Dominguez J, Lobato RD, Rivas JJ, et al: Changes in systemic blood pressure and cardiac rhythm induced by therapeutic compression of the trigeminal ganglion. Neurosurgery 43:422-428, 1994

17. Hopkins CS: Bradycardia during neurosurgery--a new reflex? Anaesthesia 43:157-158, 1988 (Letter)

18. Kerr WJ, Vance JP: Oculocardiac reflex from the empty orbit. Anaesthesia 38:833-835, 1983

19. Kleihus P, Burger PC, Scheithauer BW: Histological Typing of Tumours of the Central Nervous System. Berlin: Springer-Verlag, 1993, pp 30-31

20. Kratschmer F: [Influences of reflexes of the nasal mucosa on breathing and circulatory]. Sber Akad Wis Wien 62:147-170, 1870 (Ger)

21. Kumada M, Dampney RAL, Reis DJ: The trigeminal depressor response: a novel vasodepressor response originating from the trigeminal system. Brain Res 119:305-326, 1977

22. Lobato RD, Rivas JJ, Sarabina R, et al: Percutaneous microcompression of the gasserian ganglion for trigeminal neuralgia. J Neurosurg 72:546-553, 1990

23. Lang S, Lanigan DT, van der Wal M: Trigeminocardiac reflexes: maxillary and mandibular variants of the oculocardiac reflex. Can J Anaesth 38:757-760, 1991

24. Levy M, Martin PL: Neural control of the heart, in Berne RM, Sperelakis N, Geiger SR (eds): The Cardiovascular System. Handbbok of Physiology. Sec 1. Vol, Part1: The Heart. Bethesda, MD: 
American Physiological Society, 1979, pp 581-620

25. Loewinger J, Cohen MB, Levi E: Bradycardia during elevation of a zygomatic arch fracture. J Oral Maxillofac Surg 45:710-711, 1987

26. Menetrey D, Basbaum AI: Spinal and trigeminal projections to the nucleus of the solitary tract: a possible substrate for somatovisceral and viscerovisceral reflex activation. J Comp Neurol 255:439-450, 1987

27. Mirakhur RK, Jones CJ, Dundee JW, et al: Atropine or glycopyrrolate for the prevention of oculocardiac reflex in children undergoing squint surgery. Br J Anaesth 54:1059-1063, 1982

28. Moonie GT, Ress EL, Elton D: The oculocardiac reflex during strabismus surgery. Can Anaesthet Soc J 11:621, 1964 (letter)

29. Mullan S, Lichtor T: Percutaneous microcompression of the trigeminal ganglion for trigminal neuralgia. J Neurosurg 59:1007-1012, 1983

30. Negrutiu S, Pop R, Cardan E: [Persistent oculocardiac reflex following a retrobulbar hematoma.] Rev Chir 37:309-310, 1988 (Rom)

31. Pfaltz CR, Gratzl O: The retrosigmoid approach in cerebellopontine angle surgery: otological procedure and outcome, in Tos M, Thomsen J (eds): Acoustic Neuroma. Proceedings of the First International Conference on Acoustic Neuroma. Amsterdam: Kugler, 1992, pp 423-427

32. Precious DS, Skulsky FG: Cardiac dysrhythmias complicating maxillofacial surgery. Int J Oral Maxillofac Surg 19:279-282, 1990

33. Ragno JR Jr, Marcoot RM, Taylor SE: Asystole during Le Fort I osteotomy. J Oral Maxillofac Surg 47:1082-1083, 1989

34. Rivard JC, Lebowitz PW: Bradycardia after alfentanil-succinylcholine. Anaesth Analg 76:907, 1988 (Letter)

35. Robidaux V: Oculocardiac reflex caused by midface disimpaction. Anesthesiology 49:433, 1978 (Letter)

36. Ruggiero DA, Ross CA, Reis DJ: Projections from the spinal trigeminal nucleus to the entire length of the spinal cord in the rat. Brain Res 225:225-233, 1981

37. Schmeling WT, Kampine JP, Warltier DC: Negative chronotropic actions of sufentanil and vecuronium in chronically instrumented dogs pretreated with propanolol and/or diltiazem. Anaesth Analg 66:4-14, 1989

38. Schwartz H: Oculocardiac reflex: is prophylaxis necessary? in Mark LC, Ngai SH (eds): Highlights of Clinical Anaesthesiology. New York: Harper \& Row, 1971, pp 111-114

39. Shearer ES, Wenstone R: Bradycardia during elevation of zygomatic fractures. A variation of the oculocardiac reflex. Anaesthesia 42:1207-1208, 1987

40. Shelly MP, Church JJ: Bradycardia and facial surgery. Anaesthesia 43:422, 1988 (Letter) 
41. Spaziante R, Cappabianca $\mathrm{P}, \mathrm{Peca} \mathrm{C}$ et al: Subarachnoid hemorrhage and "normal pressure hydrocephalus": fatal complication of percutaneous microcompression of the gasserian ganglion. Neurosurgery 22:148-151, 1988

42. Starr NJ, Sethna DH, Estafanos FG: Bradycardia and asystole following rapid administration of sufentanil with vecuronium. Anesthesiology 64:521-523, 1986

43. Stott DG: Reflex bradycardia in facial surgery. Br J Plast Surg 42:595-597, 1989

44. Tenney SM, Boggs DF: Comparative mammalian respiratory control, in Fishman AP, Cherniak NS, Widdicombe JG (eds): Handbook of Physiology, Sec 2. The Respiratory System. Vol 2, Part 2:

Control of Breathing. Bethesda, MD: American Physiological Society, 1986, pp 833-855

45. Terui N, Numao Y, Kumada M, et al: Identification of the primary afferent fiber group and adequate stimulus initiating the trigeminal depressor response. J Auton Nerv Syst 4:1-16, 1981

Manuscript received July 20, 1998.

Accepted in final form August 14, 1998.

Part of this work was presented at the Joint Meeting of the Neurosurgical Society of Australasia and the Swiss Society of Neurosurgery, St-Luc, Switzerland, July 2-4, 1997; and at the Third Congress of the European Association for Neuro-Oncology, Versailles, France, September 13-16, 1998.

Address reprint requests to: Rudolf Probst, M.D., Department of Otolaryngology, University Hospitals Basel, CH-4031 Basel, Switzerland. 